\title{
Strength and Weaknesses of Cerebrospinal Fluid Biomarkers in Alzheimer's Disease and Possible Detection of Overlaps with Frailty Process
}

\author{
Alessandro Stefani ${ }^{*}{ }^{1,2}$, Enrica Olivola ${ }^{1}$, Mario Stampanoni Bassi ${ }^{1}$, Valerio Pisani, Paola Imbriani ${ }^{1}$, \\ Antonio Pisani $^{1,2}$ and Mariangela Pierantozzi ${ }^{1,2}$ \\ ${ }^{I}$ UOSD Parkinson, University of Rome Tor Vergata, Rome, Italy \\ ${ }^{2}$ IRCCS Fondazione S Lucia, Rome, Italy
}

\begin{abstract}
With the increase of human lifespan and refinement of diagnostic techniques dementia, and Alzheimer's disease (AD) in particular, have become a multi-decade process with a complex pathogenesis. The prognosis of AD patients, especially in late stages, may be strongly influenced by factors that go far beyond the well-recognized cascades (tau deposition, amyloid plaques). In this context, $\mathrm{AD}$ and Frailty, a multidimensional process of the elderly, inevitably overlap. Not surprisingly, the routine biomarkers collectable in the cerebrospinal fluid, while highly relevant in allowing specific diagnoses, becoming limiting when used to define severity and rate of progression of cognitive impairment. In reviewing merits and pitfalls of routine cerebrospinal fluid profile for $\mathrm{AD}$, this manuscript will examine the state-of-theart related to a parallel field, the extrapyramidal disorders. For synucleinopathies, we will discuss the possibility to detect factors directly involved in earliest disease pathology (alpha-synuclein, tau-proteins) together with indexes of disease progression (i.e. dopamine-metabolite ratio and loss of blood-brain barrier integrity). This approach might guarantee the capability of monitoring putative disease-modifying strategies. However, we will show the likelihood that nonconventional approaches already proposed for Frail subjects (such as exercise-mediated neuro-protection) might prove to be a useful aid for an ageing brain already impaired by AD alterations. A crucial test for these hypotheses would be to apply this sort of interventional, and not merely pharmacological, therapy to homogeneous patient cohorts.
\end{abstract}

Keywords: Ageing, cognition, cerebrospinal fluid biomarkers, dementia, frailty, neurodegenerative disease.

\section{ALZHEIMER'S DISEASE AND FRAILTY: A COMMON PROFILE OF THE AGEING BRAIN?}

The history of dementia is markeded by some paradigmatic pictures, including the sorrowful face of Auguste D, as it appeared in the impressive photograph dated November 1902. As confirmed by the historical recap published in Lancet in 1997 [1], the clinical history of this first Alzheimer's disease (AD) patient was characterized by reduced memory and comprehension, aphasia, disorientation, unpredictable behavior, paranoia, auditory hallucinations, and psychosocial impairment; a presumed four-year disease duration, and an early death caused by septicemia due to decubitus and pneumonia. All together, these aspects highlight a dreadful clinical picture dominated, since the beginning, by "limbic" features, rapid clinical deterioration and devastating complications. In the next few years following the noteworthy presentation of the Aguste D. case, given by Alzheimer at the $37^{\text {th }}$ Conference of South-West German Psychiatrists in Tubingen (1906), Perusini together with Kraepelin (who attributed the disease's eponymous to Alzheimer) re-examined the case. In the light of the clinical symptoms and histopathology findings reported by Alzheimer himself, they indicated as possible markers of "a serious form of dementia", both the abundance of plaques ("excessively numerous") and the extensive cell death

*Address correspondence to this author at the Department of Neuroscience, University of Rome "Tor Vergata", Viale Oxford 81, Rome, Italy; Tel: +3920903113; Fax: +3920903118; E-mail: stefani@uniroma2.it ("almost one/third of cortical neurons had died off") (excerpts are drawn from the handbook of Psychiatry 1910, as quoted in Lancet 1997).

Nowadays, these historical data may be re-interpreted as suggestive of a possible fragile condition, as the most advanced extremity-of-the curve of a disease's severity. Actually, in the past decades, frailty has been largely identified with disability and/or co-morbidity, but more recently, it has been recognized as a distinct clinical syndrome [2-6]. In fact, it is now well accepted that frailty represents a dynamic process of increasing vulnerability, resulting from the decline of the homeostatic reserve with a decreased ability to withstand stress, that inevitably leads to progressive destruction of multiple physiological domains, including muscle strength, balance, mobility, nutrition, physical endurance and, most notably, mood and cognition [7-11]. Therefore, since increasing evidence indicates the impact of cognition on the decline in functional capacities of the elderly, cognitive performance should be always included in the working definition of frailty.

On the other hand, it is important to outline the borders and the possible overlaps between the development of cognitive impairment and a possible underlying frailty, since both processes prevail in old age, and the latter may dramatically influence the outcome of the first one, contributing to a sort of rapid progression from delusion, loss of orientation, apraxia and other cognitive and behavioral symptoms to a full dependence from the caregiver, hospitalization, institutionalization and death. 
Nowadays, we are usually witnessing dementia progression since the earliest stage, giving relevance to a timely identification of memory complaints with the aim to achieve a clear-cut early diagnosis $[11,12]$. Several factors have repositioned the dementia time-span at least 10 years in advance: 1) the possibility to define even a slight degree of cognitive impairment; 2 ) the profuse availability of multiple neuroimaging tools (both morphometric and functional radiotracers; 3) the possibility to critically evaluate the comorbidities, such as vascular burden, through innovative biomarkers.

Of course, the likelihood of an excessive use of diagnostic tools is present, since the relatively low costs of neuroimaging have made these techniques more tolerable for public or insurance-based healthcare. There is a paradoxical frequent utilization of both magnetic resonance imaging and functional nuclear imaging over the years also in those old patients whose cognitive profile is attributable to the socalled Mild Cognitive Impairment (MCI) that may develop toward $\mathrm{AD}$, althoughcommonly remaining stable for years.

Our point, here, is that: i) an easier access to radiological tools does not necessarily mean a more accurate diagnosis; ii) in the absence of a clear estimation of clinical follow-up, even the determination of beta-amyloid peptide $(A \beta)$ or tauproteins in cerebrospinal fluid (CSF) may be not definitive; iii) current pharmacological treatments remain somehow elusive, and difficult to tailor to each individual patient. For instance, if early deterioration of synaptic modulation occurs, as the original trait of neurodegenerative diseases, years or even decades before clinical presentation, which diagnostic attitude should be used? And, more important for the aim of this issue, what really separates a pathological process from the aging-related ones?

On the other extremity of the lifespan, given both society's empathy and the excellence of the healthcare system in several countries, dementia can last for years, reaching more severe stages. Thus, which conditions or processes may actually influence prognosis, duration and quality of life in these advanced AD patients, characterized by severe loss of functional independence and abilities? Indeed, it is likely that development and time-course of dementia may be not purely ascribed to amyloid pathogenesis. It is also probable that disease symptom progression, complications and quality of life in AD patients may be related to their global state and functional reserve capacity. Recent clinical studies investigated particular forms of $\mathrm{AD}$, with "malignancy" characteristics, presenting a rapid disease progression rate, relevant extrapyramidal signs, poor or absent response to pharmacological treatment and high risk of mortality $[14,15]$. This unusual clinical picture suggests that these patients could be "frail". Moreover, the heterogeneity of AD presentations clearly indicates the need to characterize different sub-forms of disease and suggests a need to investigate the existence of frailty process among these patients. For this purpose, as reported in a different chapter of this issue, Martorana and colleagues investigated a range of markers useful to define frailty among $\mathrm{AD}$ patients. Therefore, clinical (mini-mental state exam, presence of apathy, hypertension, diabetes, hyperlipidemia), biochemical (CSF biomarkers and serum inflammatory markers like fibrinogen, c-reactive protein and D-dimers), and genetic (Apo E) factors were examined in patients with a "malignant form of AD" and matched to patients with a more regular response to treatment and a low rate of disease progression. The results, despite extremely high levels of total $(\mathrm{t})$-tau may characterize $\mathrm{AD}$ patients with more rapid cognitive worsening, thus pointing to the need to indentify more reliable ones [16].

\section{STRENGTHS AND LIMITATIONS OF CURRENTLY AVAILABLE CSF BIOMARKERS IN AD}

As already mentioned in the previous chapter, in the last decades we have witnessed a profound revision of the dementia background that allows one to substantiate early diagnosis of AD using both radiological and biochemical markers. This provides a rationale for large-scale trials with agents devoted to the hypothetical rescue of cognitive and functional abilities. However, as diagnostic capabilities approach the neurodegenerative process critical issues remain. On the one hand, efficacious neuroprotective agents remain to be identified; on the other, we still struggle to find tools able to identify the more at-risk subjects, namely those who are more prone to develop a rapid or "malignant form of AD". A recent longitudinal study evaluated the 5-year survival rate in a cohort of $\mathrm{AD}$ patients, which had undergone, at baseline, cognitive testing and CSF biomarker assessment [14]. The results suggested that a small cohort $(8 \%)$ of patients, having low baseline levels of $A \beta$ but very high levels of both t-tau and phosphorylated ( $\mathrm{p}$ )-tau proteins and worse cognitive tests, exhibited a very poor response to cholinesterase inhibitor treatment, and where "cognition deteriorated faster over time and the mortality rate substantially increased". Yet, these findings are not entirely convincing. In fact, although currently available CSF biomarkers can be properly used in the assessment of large cohorts of AD patients, they are still neither exhaustive, nor completely specific as a diagnostic tool for discerning different subtypes of dementia. Their utilization, indeed, suffers from several pitfalls especially in those AD patients with atypical presentation, including abundant extrapyramidal signs, which may lie on the border between $\mathrm{AD}$ and Lewy body disorders. Such ambiguity can occur also when there is a request to obtain a clear picture about whether and when an individual will experience a steep deterioration or, finally, which rate of progression will characterize the time-course of the disease.

The above caveats notwithstanding, CSF biomarkers deserve their just consideration. It is unquestionable that their introduction has offered neurologists an instrument to investigate the involvement of amyloid precursor protein metabolism and tau-proteins in the pathophysiology of AD, and to promote the development of drugs affecting the underlying disease pathophysiology $[17,18]$. Moreover, the possibility to determine their concentrations in a patient's CSF allows one to avoid misdiagnoses. As increasingly recognized, and recapped by Stefani and co-authors [19] CSF biomarkers, specifically the t-tau/A $\beta$ ratio are decisive to make a diagnosis of AD in early phases of disease. This last point is important when consistent clinical information is difficult to collect (or social conditions hamper their validation), or when an early pseudo-psychic presentation 
prevails or even in case of conversion from MCI to early AD. Thus, they contribute to the ante-mortem identification of $\mathrm{AD}$, with a fairly safe and low-cost approach. However, the success and the commercial diffusion of these diagnostic tools should not under-evaluate limits. In this respect, an enlarged multicentre study tried to improve the accuracy of CSF biomarkers, reducing inter-site assay variability amongst laboratories in different countries and highlighting the necessity for standardization of analytical techniques [20].

A summary of the main pros and cons related to the use of currently available CSF biomarkers in dementia is highlighted in Table 1, which tries to synthesize, albeit in a heuristic manner, our feelings.

In other words, the most relevant CSF biomarker limits, as summarized in Table 1, are the following: weak ability to provide stage disease assessment; namely, which kind of CSF biomarkers have to be considered and/or correlated for in fieri deterioration? Most studies describe a fixed picture taken in a clinical momentum. How many studies monitor CSF biomarkers along with the clinical/cognitive evolution (or radiological changes) of disease? Unfortunately few: for instance, Siderowf and co-authors [38], studying the cognitive decline associated with Parkinson's disease (PD), suggested that reduced CSF A $\beta$ might be considered as independent predictor of cognitive decline in these patients. In fact they found that non-demented PD patients showing at baseline the lowest CSF A $\beta$ concentration developed the greatest decline in attention, conceptualization and memory at the next follow-up. On the other hand, detection of longitudinal changes in CSF levels of AD biomarkers, and their possible relationship with progression of a patient's cognitive decline was investigated by performing two lumbar punctures, at a median interval of 3 years, in the same subjects categorized as MCI, AD or affected by other neurological disorders [39]. This approach allowed the assessment that $\mathrm{AD}$ patients displaying the most rapid
MMSE decline rate had, at baseline, the lowest $A \beta$, highest t-tau, and highest p-tau-181 CSF contents. Moreover, AD patients presented an annual reduction of $2.20 \mathrm{pg} / \mathrm{ml} / \mathrm{year}$ in the CSF p-tau-181 concentration, which was significant if compared to stable MCI and cognitively healthy subjects. Finally, in AD patients the decrease rate of p-tau-181 correlated with MMSE. These results need to be confirmed and extended even in patients at the preclinical stage of disease, but do many longitudinal studies of CSF biomarker dynamics currently exist? Not many, to our knowledge. In this respect, a critical aspect consists of when CSF samples should be collected. So far, ethical or bureaucratic reasons (last but not least, the resistance of the traditional academic milieu, still refusing to include in the clinical guidelines the assessment of CSF as a reliable tool) relegate the execution of lumbar puncture as a research approach, which has to be established in advance, then planned with the patient and the caregiver, and finally performed when possible. Hence, CSF collection in the overwhelming majority of cases is not performed at the time of a specific acute derangement of cognitive performance. In other words, our clinical practice per se contributes to maintain a non-specific value to these indices. Given that, it is not generally envisioned the chance to identify a peculiar CSF profile related to the critical phases of a dementia's history. Recently it has been hypothesized that AD patients with an elevated titer of t-tau (in the 800-1200 pM range) develop an unusually fast and malignant disease $[14,16]$.

\section{AN EXEMPLARY ANGLE OF VISION: EXTRA- PYRAMIDAL SYNDROMES}

Taking into account to what extent a different rate of disease progression may dramatically affect a patient's clinical history, it is unquestionable that a better understanding of the pathogenetic mechanisms underlying the time-course of neurodegenerative diseases is of primary importance in clinical practice and research as well. In this

Table 1. Essential Advantages and Pitfalls Related to the Use of CSF Biomarkers in Dementia

\begin{tabular}{|c|c|}
\hline $\begin{array}{l}\text { corroborate AD diagnosis in early phases } \\
\text { especially versus pseudo-dementia } \\
\text { Dubois et al.,2010 [21]; Wagner et al., } 2012 \text { [22] }\end{array}$ & $\begin{array}{l}\text { lack of specificity versus AD with extra-pyramidal involvement and DLB } \\
\text { Compta et al.,2009 [23]; Montine et al.,2010 [24]; } \\
\text { Parnetti et al., } 2010 \text { [13]; Prrikrylová et al., } 2010 \text { [25]; } \\
\text { Schoonembomm et al., } 2012 \text { [26] }\end{array}$ \\
\hline $\begin{array}{l}\text { clarify diagnosis in previously defined } \\
\text { "mixed" dementia } \\
\text { (i.e. with extensive vascular burden) } \\
\text { Stefani et al., } 2005 \text { [30]; Gomis et al., } 2009 \text { [31]; } \\
\text { Goos et al., } 2011 \text { [32] }\end{array}$ & $\begin{array}{l}\text { low sensitivity in most of cases } \\
\text { of small vessel disease } \\
\text { Carmichael } \text { et al., } 2010 \text { [33]; } \\
\text { Viswanathan and Greenberg, } 2011 \text { [34] }\end{array}$ \\
\hline good correlation with familial forms & $\begin{array}{l}\text { insufficient findings in the extreme old patients } \\
\text { Vemuri et al., } 2011 \text { [35]; Schneider et al., } 2009 \text { [36]; Kester et al., } 2010 \text { [37]; }\end{array}$ \\
\hline
\end{tabular}


perspective, a realistic goal may be represented by the possibility to distinguish different "disease subtypes", which may differ in terms of pathogenesis and prognosis. In the last years, several biochemical, neuropsychological, electrophysiological and neuro-radiological tools have been investigated as possible reliable markers of disease progression. In order to develop innovative and diseasemodifying strategies, the combined acquisition of different markers with adequate specificity and sensitivity in monitoring the disease evolution is needed. In this framework, PD provides an interesting example. Due to the large amount of data collected in last decades, the definition of the disease itself, traditionally corresponding to an exclusive movement disorder, was modified to the concept of a complex multisystem disease, featured by different nonmotor profiles including, cognitive, mood, sleep and behavior disturbances [40]. According to this, dementia in $\mathrm{PD}$ is no longer considered a mere complication of the most advanced stages. It may affect, albeit insidiously, the early phases of disease, and characterize specific disease subtypes. For instance, several lines of evidence indicate that in PD patients, severe cognitive impairment strongly correlates with axial deficit. Among the others, Alves and coauthors showed that "in a substantial proportion of PD patients who develop postural instability and gait disorder during the course of the disease, this transition was associated with accelerated cognitive decline and highly increased risk for subsequent dementia", whilst "patients with Tremor Dominant subtype at baseline did not become demented until they developed Postural Instability Gait Disturbances subtype, and dementia did not occur among patients with persistent Tremor Dominant subtype of Parkinsonism" [41]. Moreover, even if pathologists still indicate the "inevitability of dementia" in the 15-20 years of PD history frame (and less then $52 \%$ in ten years) $[42,43]$, severe cognitive derangement cannot be excluded in early phases of PD, but its actual occurrence remains quite doubtful. In keeping with these novel findings, the CSF biomarkers typically utilized in $\mathrm{AD}$ were also tested in PD patients, in order to understand if and to what extent $A \beta$, t-tau, $\mathrm{p}$-tau, and tau/amyloid ratio correlate with specific clinical presentations of extrapyramidal disorders, including PD, PD with dementia (PD-D) and dementia with Lewy bodies (DLB). This scenario, as recently reviewed by Stefani and coworkers [19], appears still complex. However, it is fairly evident that the identification of different CSF biomarker profiles, combined to functional neuro-imaging, and targeted neuropsychological assessments, may be helpful in distinguishing different subgroups of DLB, depending on the presence or absence of a relevant amyloid burden. On the other hand, the fact that current diagnostic tools are still inappropriate makes it necessary to search for novel and more appropriate disease biomarkers. In fact, for clinically diagnosed DLB cases, although amyloid pathology is strongly suspected, unequivocal evidence on the possible mechanisms involved in the neuronal degeneration has not been fully achieved so far. As a matter of fact, low A $\beta$ levels may be detected in CSF of most of DLB patients; however, also PD patients turning into PD-D could manifest a low tau/amyloid ratio in advance [44]. Yet, the finding that in a large cohort of patients with different types of dementia, a CSF AD biomarker profile was seen in $47 \%$ of DLB patients, confirms once more the need to identify additional disease biomarkers [26]. Similarly, several studies found AD-like CSF biomarker patterns in PD-D patients, but whether this means an association between $\mathrm{PD}$ and $\mathrm{AD}$ or, rather identifies a cognitive decline due to $\mathrm{PD}$ remains unknown [45]. Not surprisingly, the guidelines of most neurological societies still consider the timing criteria as the most certain for the diagnosis of DLB compared to PD-D, at least until reliable clinical, biochemical or morphologic tools become available.

This view is even more complicated by the observation that $\mathrm{AD}$ patients, whose clinical presentation is characterized by extrapyramidal signs, may fall into the ambiguous category of "malignant form of AD", which may include both MCI converted and AD with rapid disease progression. A recent clinical review suggested four prototypical AD presentations and supported the contention by which the early onset of parkinsonism and myoclonus predicts a rapid evolving of cognitive impairment, and a more severe rate of disease progression with psychiatric disorders and dependency in daily living activities [15].

The limited number of investigations linking CSF biomarkers with anatomical-pathological findings still remains a major hindrance. Recent neuropathological studies have described widespread $A \beta$ deposition in the striatum of patients with DLB and PD-D. A prospective study carried out on 34 DLB and PD-D patients showed striatal A $\beta$ immunoreactive plaques only in DLB. In these cases striatal A $\beta$ deposition correlated with both severity (positive correlation) and duration (negative correlation) of dementia. These data suggest that striatal $A \beta$ deposition in Lewy body disorders (LBDs) contributes to the occurrence of early dementia, and may impact on the efficacy of treatments targeting the striatum [46]. Moreover, a different study found a greater striatal $\mathrm{A} \beta$ deposition in DLB and PD-D, when compared to $\mathrm{PD}$, multiple system atrophy and progressive supranuclear palsy, thus suggesting that striatal $A \beta$ pathology may reflect the development of dementia in DLB and PD-D, as well [47]. Following the hypothesis that PD and $\mathrm{AD}$, traditionally viewed as distinct clinical and pathological entities, may have intriguing overlaps, the possibility to realize post-mortem exams in LBDs patients, including PD-D, DLB and PD, should provide relevant clues to confirm that "substantial bridges between taupathies and synucleinopathies occur" [48]. In this respect, it is interesting to mention that mutations in LRRK2, a well-known cause of a genetic form of parkinsonism with dopaminergic neuronal degeneration, may be associated "with the accumulation of synuclein, tau, neither, or both proteins" [48].

The aforementioned considerations underline, even further, the need to have reliable markers for the pathogenesis of neurodegenerative diseases, especially if multiple functions/systems are involved (i.e. motor, behavioral, cognitive).

In keeping with the search for relatively inexpensive and reliable biomarkers, which could detect neurodegeneration before irreversible steps have occurred, an extensive body of literature has investigated CSF levels of alpha-synuclein $(\alpha-$ Syn) in different LBDs and AD, as well. 
Recent studies have demonstrated that $\alpha$-Syn can be detected in humans CSF with a high degree of uniformity by means of enzyme-linked immunosorbent assay, and that CSF $\alpha$-Syn levels are significantly lower in all LBDs, when compared to controls. Tokuda and coauthors reported that PD patients have significantly lower $\alpha$-Syn levels in the CSF than controls with an inverse correlation between CSF $\alpha$-Syn content and the Hoehn and Yahr clinical scale, thus promoting this protein as a possible useful laboratory biomarker for PD diagnosis. The reduced CSF $\alpha$-Syn levels in PD patients with a long illness duration was interpreted as the result of progressive intracellular aggregation and accumulation of $\alpha$-Syn in the brain [49]. Nevertheless, other authors raised the question of a possible low specificity of $\alpha$ Syn as a neurodegenerative disease biomarker in distinguishing PD from other atypical parkinsonisms [50]. Furthermore, a different investigation reported that $\mathrm{PD}$ and DLB patients had similar CSF $\alpha$-Syn levels compared to controls, whereas significantly lower levels of $\alpha$-Syn were found in AD patients with respect to normal subjects; the $\alpha$ Syn content in AD was also associated with disease severity. These data clearly "speak against CSF $\alpha$-synuclein as a reliable biomarker for PD and DLB". The findings of lower CSF $\alpha$-Syn levels in AD, and the association of such a decrease with AD severity, as stated by mini mental state exam, led the authors to suggest that $\alpha$-Syn may be a general marker of synapse loss. There is no doubt that this hypothesis needs further investigation [51].

In this complex framework, it is still conflicting whether CSF $\alpha$-Syn levels clearly differ between LBDs and AD. Several authors found that $\alpha$-Syn quantification in CSF may be helpful in differentiating synucleinopathies from $\mathrm{AD}$, since CSF $\alpha$-Syn measured in advanced PD, multiple system atrophy and DLB was consistently lower in comparison to $\mathrm{AD}[52,53]$. It has also been reported that $\alpha$-Syn levels did not significantly differ between DLB and AD, but the same authors showed that, in contrast to $\mathrm{AD}$, in DLB patients the disease duration was correlated to lower $\alpha$-Syn, probably due to increased severity of $\alpha$-synucleinopathy in the brain [54]. Nevertheless, despite the certain value of $\alpha$-Syn as new CSF biomarker, as discussed by Parnetti and colleagues: " $\alpha$-Syn alone did not provide relevant information for PD"; a superior performance emerged from the combined use of $\alpha$ Syn coupled with classical neurodegenerative biomarkers, in particular the t-tau/ $\alpha$-Syn ratio and $p$-tau $/ \alpha$-Syn ratio could contribute to the identification of differential CSF patterns in neurodegenerative disorders [55]. Accordingly, Shi and colleagues, moving from the evidence that a decreased DJ-1 and/or $\alpha$-Syn content in CSF may represent an index for PD diagnosis, but not for PD severity, suggested to use a battery of additional peptides measuring t-tau, $\mathrm{p}$-tau, $\mathrm{A} \beta$, Flt3 ligand, and fractalkine [56]. These data, once again, confirm that the combined determination of multiple biomarkers, such as $\alpha$ Syn, the classical tau-proteins and new peptides in CSF may help us identify specific CSF patterns related to different neurodegenerative disorders, and this might be useful for differential diagnosis and correlation with disease progression.

At least for motor disorders, our group has consistently pursued the goal to identify CSF markers for early diagnosis and disease progression. Therefore, we have studied the CSF concentration of dopamine (DA) metabolites in PD patients through lumbar puncture, either in basal condition or under a challenge dose of levodopa. If, as hypothesized, the decline in rate of endogenous DA metabolism affects not only the motor presentation of $\mathrm{PD}$, but also cognitive performance, there is a chance that determination of CSF DA metabolites may become an important diagnostic tool for detecting initial cognitive impairment in these patients. Decades later from the first pioneering experience dealing with catecholamine

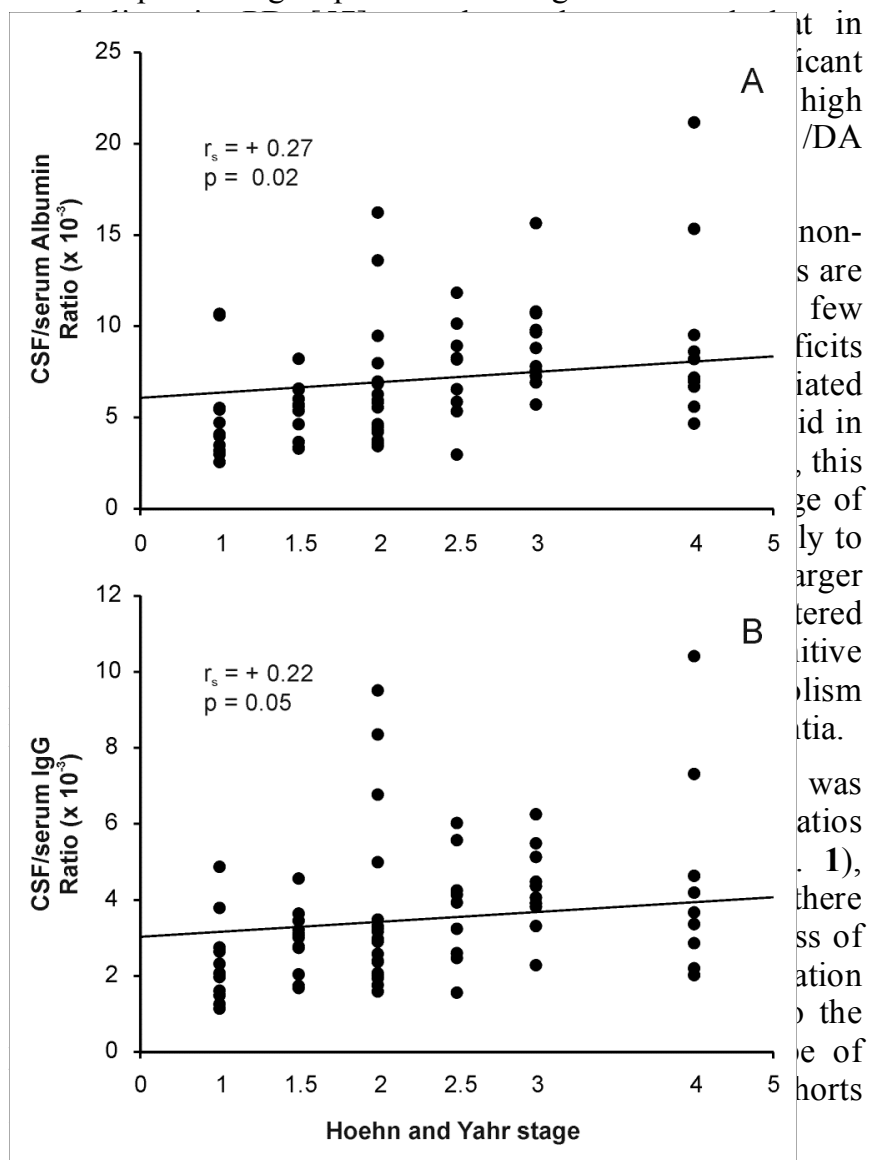

Fig. (1).

\section{FRAILTY AND AD; A COMMON STRATEGY?}

As we have remarked, the elongation of our lifespan, due to both preventive therapies and achievement of adequate care for the elderly, is allowing to follow-up patients, suffering from dementia, to live a long period of time up to the terminal stages of disease. In addition, since most of the individuals express the wish (or the anxiety?) to get as early as possible a proper diagnosis for even slight memory complaints, dementia (with $\mathrm{AD}$ encountering for $>$ than $50 \%$ ) has turned into a multi-decade process.

$\mathrm{AD}$ and frailty might represent two different aspect of the same scenario, since both processes prevail in old age. Consequently, overlaps between cognitive impairment and an underlined vulnerability can be always considered in the elderly. Frailty represents amplification and anticipation of processes superimposed with ageing. Of course, AD per se would accelerate a frail state, and frailty itself would influence functional activities of demented subjects. But which role is played by naturally occurring senescence? The 
recent availability of functional magnetic resonance imaging showed that activity changes in the hippocampus can distinguish normal ageing, featured by reduced metabolism in the subiculum and dentate gyrus, from pathological alterations in incipient $\mathrm{AD}$, where a reduced metabolism is recognizable in the entorhinal cortex. Yet, is it not true that synaptic loss, deposition of amyloid plaques, loss of volume of cortical gyrus, and not only of temporal cortices, are conditions which could be variably present in any senescence process [60]?

However, multiple, additional mechanisms come into play, as far as a huge variability of compensatory processes occurs in ageing brain. The capability of resisting transient or chronic stress might derive from a sort of "controlled" loss of mitochondrial functions (contributing to longevity and not necessarily facilitating dementia!). Yet, in frail subjects, more persistent stress might further impair mitochondrial activity up to a "self-reinforcing cycle of detrimental decline" [61]. Furthermore, a global loss of integrative function characterizes ageing brains, together with the occurrence of less specialized localization in response to executive level tasks. But again, which role is exerted by "suppletive" cortical regions in ageing $v s$ demented brains?

Today there is an urgent need to update current CSF biomarkers. If the most important aim is to achieve a personalized therapy, tailoring it on individual patients or, at least, on specific sub-cohorts, we should define additional biomarkers, possibly correlated to ageing, gender, education, and life style. Take, for example two women: the first one (in her sixties), manifesting apraxic and anomic AD, with early loss of insight, is the daughter of an $\mathrm{AD}$ patient who died at eighty because of dementia marasmus. The other one, already suffering from borderline hypertension and afflicted by early arthritis with a recent hip fracture, which halted her rehab program, is experiencing, since 3-4 years a slowly progressive cognitive deterioration, with delusions and social retirement. Noteworthy is that both of them have quite similar concentrations of $\mathrm{p}$-tau and analogous $\mathrm{t}$-tau/A $\beta$ ratio in their CSF. Would they be condemned to the same disease profile? Now consider two male AD patients both 71 years old, lacking apparent familiar traits and both experiencing memory complaints since the age of 69 . The first one has lived isolated in the mountains, working as a farmer, with little opportunity to talk and little social life. The second one, instead, is still a productive manager in a financial company. He continues to maintain an adequate physical activity associated to a balanced diet; yet, family members recognized behavioral symptoms, including apathy. Both patients share similar positron emission tomography findings and entorhinal atrophy plus analogous CSF biomarker profiles. Yet, despite these similarities, they are probably entitled to very different prognosis!

The opportunity to acquire a broader set of biomarkers is of paramount importance if we consider future tasks, as the definition of more complex therapies, as synthesized by Crews and Mesliah, when they state that "new approaches are currently being tested, including gene therapy, vaccination, changes in lifestyle that enhances neurogenesis, intra-thecal drug delivery and use of compounds bound to lipids" [61].
The presently available CSF biomarkers reveal their limits, especially when applied to community surveys. Not surprisingly, Haldenwanger and co-authors, in a study devoted to the spectrum of MCI-AD patients, which was performed in a non-academic memory clinic, investigated to what extent CSF findings did in fact contribute to confirm or modify clinically-based diagnoses. After CSF biomarker levels were revealed, only 11 diagnoses changed. "The knowledge of CSF biomarker profiles changed the diagnosis in $10 \%$ of the cases, and confidence in the diagnosis increased for one third of the patients" [29]. Hence, community studies on a homogeneous AD population (i.e., the old nun study! [62]) are welcome, with the purpose to match not only age and gender, but also education, social habits, life style and variety of sensory inputs.

The available literature on frailty allows an interesting digression. Classical studies on frailty have proposed already that, in relation to the decline in homeostatic reserves, 3 stages can be described: a pre-frail process, the frailty state and frailty complications [63]. This, somehow in analogy with $\mathrm{AD}$, confirms that frailty is a progressive condition, beginning with a preclinical stage, thereby allowing room for early prevention [3].

Noteworthy, it was inferred that it is not satisfactory to define frailty in the physical domain alone, since several other domains, first of all cognition, are widely recognized as part of the frailty state. Frailty state is largely characterized by other components of the syndrome such as mood, cognition, sensory impairments and socioeconomic aspects of older adults' lives (again overlapping with dementia) $[2,64]$. Among the ways to fight against frailty, as suggested by Leber [65], one should consider "1) adequate diet with sufficient protein, vitamin and mineral intake; (2) regular physical exercise, practiced alone or in groups, such as stretching, walking, dancing, dynamic balance exercise and lifting weights; and (3) rapid reconditioning after stressful events via re-nutrition and individually tailored physiotherapy". Consistently, in a randomized controlled trial, conducted on 150 sedentary community-dwelling men and women aged 78 years or older with mild to moderate physical frailty, Binder et al. [66] assessed the effect of exercise training on frailty. The results were very impressive, supporting the possible reversibility of functional decline in the exercising group. In a second controlled study, Gill et al. randomly assigned 188 older adults of 75 years or more (physically frail and living at home) to undergo a 6-month, home-based intervention program. The latter included "physical exercise therapy" and focused "on improving underlying impairments in physical abilities, including balance and muscle strength, the ability to transfer from one position to another and mobility". Unfortunately, "the benefit of intervention was observed among participants with moderate frailty and not those with severe frailty". However, this home-based program confirmed the ability to reduce the progression of functional decline among physically frail elderly people [67]. Whether also progressive cognitive deterioration in $\mathrm{AD}$ patients may be preventable or, more realistically, slowed by a similar approach, is a matter of constant investigation.

Although data from intervention-based randomized trials are scarce, there is some indication that exercise may protect 
against age-related deficits in cognitive function. "Data from animal models suggest that exercise, in the form of voluntary wheel running, is associated with reduced amyloid deposition and enhanced clearance of $\mathrm{A} \beta$, the major constituent of plaques in AD. A common therapeutic theme arising from studies of exercise-induced neuroprotection in human populations and in animal models involves reduced inflammation in the CNS. In this respect, physical activity may promote neuronal resilience by reducing inflammation" [68]. Not surprisingly, similar data are emerging also for cognitive impairment among extra-pyramidal disorders [69].

\section{CONCLUSIONS}

- $\quad$ Expansion of lifespan, together with understanding some of the core pathogenetic cascades underlying cortical dementias has revolutionized our concept of AD: from a dramatic disease covering 5-10 years to a 3-5 decade process. An illuminated healthcare, considering that nearly $50 \%$ subjects 85 years old are going to be affected by $\mathrm{AD}$, should envision which factors determine disease progression and impose full disability.

- Despite similar biochemical events taking place in the vast majority of $\mathrm{AD}$ patients (as revealed also by CSF samples), the clinical duration and profile may vary dramatically, especially in late life stages and/or lateonset forms. A previous point of view suggested that low education levels, younger onset, presence of hallucinations or extrapyramidal signs, low CSF A $\beta$ 42, very high $t$ - tau or $p$-tau levels and high tau/A $\beta_{s}$ ratio, were predictors of a worse disease progression rate. But, in our belief, and as summarized in this manuscript, such a combination is not exhaustive or sufficient.

- Frailty goes far beyond a loss of metabolic homeostasis and includes a vicious cycle comprising cognitive impairment, lack of caregiving, comorbidities and social retirement (which goes with apathy and loss of physical exercise). Emerging evidence is highlighting a subgroup of patients, among $\mathrm{AD}$, exhibiting a peculiar frailty and poor response to therapy.

- $\quad$ Our therapeutic armamentarium is going to change; from a therapy based on molecules facilitating cholinergic transmission to strategies aiming to rescue $\mathrm{A} \beta$ or tau depositions. Furthermore, an alternative approach will include protection of selective neuronal populations, promotion of synaptic formation, and modulation of neurogenesis, but not to the exclusion of conventional techniques.

- Non-pharmacological factors, such as exercisemediated neuroprotection, are suggested to play a relevant role in plastic remodelling of the ageing brain. Consistently, it is urgent to test these hypotheses in AD cohorts, similar in gender, age, and most importantly socio-economical status and life style.
ABBREVIATIONS

$\mathrm{A} \beta=$ Beta-amyloid
$\mathrm{AD}=$ Alzheimer's disease
$\alpha-\mathrm{Syn}=$ alpha-Synuclein
$\mathrm{CSF}=$ Cerebrospinal fluid
$\mathrm{DA}=$ Dopamine
$\mathrm{DLB}=$ Dementia with Lewy bodies
LBDs $=$ Lewy body disorders
MCI $=$ Mild cognitive impairment
$\mathrm{PD}=$ Parkinson's disease
PDD $=$ Parkinson's disease with dementia
(t)-tau $=$ Phosphorylated
(p)-tau

\section{REFERENCES}

[1] Maurer, K.; Volk, S.; Gerbaldo, H. Auguste D and Alzheimer's disease. Lancet, 1997, 349(9064), 1546-1549.

[2] Fried, L.P.; Tangen, C.M; Walston, J.; Newman, A.B.; Hirsch, C.; Gottdiener, J.; Seeman, T.; Tracy, R.; Kop, W.J.; Burke, G.; McBurnie, M.A.; Cardiovascular Health Study Collaborative Research Group. Frailty in older adults: evidence for a phenotype. J. Gerontol. A Biol. Sci. Med. Sci., 2001, 56(3), M146-156.

[3] Fried, L.P.; Ferrucci, L.; Darer, J.; Williamson, J.D.; Anderson. G. Untangling the concepts of disability, frailty, and comorbidity: implications for improved targeting and care. J. Gerontol. A. Biol. Sci. Med. Sci., 2004, 59(3), 255-263.

[4] Fried, L.; Walston, J.D.; Ferrucci, L. Frailty, $6^{\text {th }}$ ed.; McGraw Hill: New York, 2009, Chapter 52.

[5] Lipsitz, L.A. Dynamic models for the study of frailty. Mech. Ageing Dev., 2008, 129(11), 675-676.

[6] Shamliyan, T.; Talley, K.M.; Ramakrishnan, R.; Kane, R.L. Association of frailty with survival: A systematic literature review. Ageing Res. Rev., 2012. [Epub ahead of print]

[7] Chin, A.P.M.J.; Dekker, J.M.; Feskens, E.J.; Schouten, E.G.; Kromhout, D. How to select a frail elderly population? A comparison of three working definitions. J. Clin. Epidemiol., 1999, 52(11), 1015-1021.

[8] Cohen, H.J. In search of the underlying mechanism of frailty. $J$. Gerontol. A. Biol. Sci. Med. Sci., 2000, 55(12), M706-M708.

[9] Dayhoff, N.E.; Suhrheinrich, J.; Wigglesworth, J.; Topp, R.; Moore, S. Balance and muscle strength as predictors of frailty among older adults. J. Gerontol. Nurs., 1998, 24(7), 18-27.

[10] Ferrucci, L.; Guralnik, J.M.; Studenski, S.; Fried, L.P.; Cutler, G.B.Jr.; Walston, J.D.; Interventions on FrailtyWorking Group. Designing randomized, controlled trials aimed at preventing or delaying functional decline and disability in frail, older persons: a consensus report. J. Am. Geriatr. Soc., 2004, 52(4), 625-634.

[11] Walston, J.; Hadley, E.C.; Ferrucci, L.; Guralnik, J.M.; Newman, A.B.; Studenski, S.A.; Ershler, W.B.; Harris, T.; Fried, L.P. Research agenda for frailty in older adults: toward a better understanding of physiology and etiology: summary from the American Geriatrics Society/National Institute on Aging Research Conference on Frailty in Older Adults. J. Am. Geriatr. Soc., 2006, 54(6), 991-1001.

[12] Heister, D.; Brewer, J.B.; Magda, S.; Blennow, K.; McEvoy, L.K.; Alzheimer's Disease Neuroimaging Iniziative. Predicting MCI outcome with clinically available MRI and CSF biomarkers. Neurology, 2011, 77(17), 1619-1628.

[13] Parnetti, L.; Chiasserini, D.; Eusebi, P.; Giannandrea, D.; Bellomo, G.; De Carlo, C.; Padiglioni, C.; Mastrocola, S.; Lisetti, V.; Calabresi, P. Performance of a $\beta 1-40, a \beta 1-42$, total tau, and phosphorylated tau as predictors of dementia in a cohort of patients with mild cognitive impairment. J. Alzheimers Dis., 2012, 29(1), 229-238. 
[14] Wallin, A.K.; Blennow, K.; Zetterberg, H.; Londos, E.; Minthon, L.; Hansson, O. CSF biomarkers predict a more malignant outcome in Alzheimer disease. Neurology, 2010, 74(19), 1531-1537.

[15] Mangone, C.A. Clinical heterogeneity of Alzheimer's disease. Different clinical profiles can predict the progression rate. Rev. Neurol., 2004, 38(7), 675-681.

[16] Koch, G.; Belli, L.; Lo Giudice, T.; Di Lorenzo, F.; Sancesario, G.; Lubrano, A.; Bernardini, S.; Martorana, A. Frailty among Alzheimer's disease patients. CNS Neurol. Dis. Drug Targets. This issue.

[17] Blennow, K. Biomarkers in Alzheimer's disease drug development. Nat. med., 2010, 16(11), 1218-1222.

[18] Blennow, K.; Hampel, H.; Weiner, M.; Zetterberg, H. Cerebrospinal fluid and plasma biomarkers in Alzheimer disease. Nat. Rev. Neurol., 2010, 6(3), 131-144.

[19] Stefani, A.; Brusa, L.; Olivola, E.; Pierantozzi, M.; Martorana, A. CSF and clinical hallmarks of subcortical dementias: focus on DLB and PDD. J. Neural. Transm., 2012, 119(7), 861-875.

[20] Mattsson, N.; Zetterberg, H.; Hansson, O.; Andreasen, N.; Parnetti, L.; Jonsson, M.; Herukka, S.K.; van der Flier, W.M.; Blankenstein, M.A.; Ewers, M.; Rich, K.; Kaiser, E.; Verbeek, M.; Tsolaki, M.; Mulugeta, E.; Rosén, E.; Aarsland, D.; Visser, P.J.; Schröder, J.; Marcusson, J.; de Leon, M.; Hampel, H.; Scheltens, P.; Pirttilä, T.; Wallin, A.; Jönhagen, M.E.; Minthon, L.; Winblad, B.; Blennow, $\mathrm{K}$. CSF biomarkers and incipient Alzheimer disease inpatients with mild cognitive impairment. JAMA, 2009, 302(4), 385-393.

[21] Dubois, B.; Feldman, H.H.; Jacova, C.; Cummings, J.L.; Dekosky, S.T.; Barberger-Gateau, P.; Delacourte, A.; Frisoni, G.; Fox, N.C.; Galasko, D.; Gauthier, S.; Hampel, H.; Jicha, G.A.; Meguro, K.; O'Brien, J.; Pasquier, F.; Robert, P.; Rossor, M.; Salloway, S.; Sarazin, M.; de Souza, L.C.; Stern, Y.; Visser, P.J.; Scheltens, P. Revising the definition of Alzheimer's disease: a new lexicon. Lancet Neurol., 2010, 9(11), 1118-1127.

[22] Wagner, M.; Wolf, S.; Reischies, F.M.; Daerr, M.; Wolfsgruber, S.; Jessen, F.; Popp, J.; Maier, W.; Hüll, M.; Frölich, L.; Hampel, H.; Perneczky, R.; Peters, O.; Jahn, H.; Luckhaus, C.; Gertz, H.J.; Schröder, J.; Pantel, J.; Lewczuk, P.; Kornhuber, J.; Wiltfang, J. Biomarker validation of a cued recall memory deficit in prodromal Alzheimer disease. Neurology, 2012, 78(6), 379-386.

[23] Compta, Y.; Martí, M.J.; Ibarretxe-Bilbao, N.; Junqué, C.; Valldeoriola, F.; Muñoz, E.; Ezquerra, M.; Ríos, J.; Tolosa, E. Cerebrospinal tau, phospho-tau, and beta-amyloid and neuropsychological functions in Parkinson's disease. Mov. Disord., 2009, 24(15), 2203-2210.

[24] Montine, T.J.; Shi, M.; Quinn, J.F.; Peskind, E.R.; Craft, S.; Ginghina, C.; Chung, K.A.; Kim, H.; Galasko, D.R.; Jankovic, J.; Zabetian, C.P.; Leverenz, J.B.; Zhang, J. CSF A $\beta(42)$ and tau in Parkinson's disease with cognitive impairment. Mov. Disor., 2010, 25(15), 2682-2685.

[25] Přikrylová Vranová, H.; Mareš, J.; Nevrlý, M.; Stejskal, D.; Zapletalová, J.; Hluštík, P.; Kaňovský, P. CSF markers of neurodegeneration in Parkinson's disease. $J$. Neural Transm., 2010, 117(10), 1177-1181.

[26] Schoonenboom, N.S.; Reesink, F.E.; Verwey, N.A.; Kester, M.I.; Teunissen, C.E.; van de Ven, P.M.; Pijnenburg, Y.A.; Blankenstein, M.A.; Rozemuller, A.J.; Scheltens, P.; van der Flier, W.M. Cerebrospinal fluid markers for differential dementia diagnosis in a large memory clinic cohort. Neurology, 2012, 78(1), 47-54.

[27] Blom, E.S.; Giedraitis, V.; Zetterberg, H.; Fukumoto, H.; Blennow, K.; Hyman, B.T.; Irizarry, M.C.; Wahlund, L.O.; Lannfelt, L.; Ingelsson, M. Rapid progression from mild cognitive impairment to Alzheimer's disease in subjects with elevated levels of tau in cerebrospinal fluid and the APOE epsilon4/epsilon4 genotype. Dement. Geriatr. Cogn. Disord., 2009, 27(5), 458-464.

[28] Stefani, A.; Martorana, A.; Bernardini, S.; Panella, M.; Mercati, F.; Orlacchio, A.; Pierantozzi, M. CSF markers in Alzheimer disease patients are not related to the different degree of cognitive impairment. J. Neurol. Sci., 2006, 251(1-2), 124-128.

[29] Haldenwanger, A.; Eling, P.; Kastrup, A.; Hildebrandt, H. Correlation between cognitive impairment and CSF biomarkers in amnesic MCI, non-amnesic MCI, and Alzheimer's disease. $J$. Alzheimers Dis., 2010, 22(3), 971-980.

[30] Stefani, A.; Bernardini, S.; Panella, M.; Pierantozzi, M.; Nuccetelli, M.; Koch, G.; Urbani, A.; Giordano, A.; Martorana, A.; Orlacchio, A.; Federici, G.; Bernardi, G. AD with subcortical white matter lesions and vascular dementia: CSF markers for differential diagnosis. J. Neurol. Sci., 2005, 237(1-2), 83-88.

[31] Gomis, M.; Sobrino, T.; Ois, A.; Millán, M.; Rodríguez-Campello, A.; Pérez de la Ossa, N.; Rodríguez-González, R.; Jiménez-Conde, J.; Cuadrado-Godia, E.; Roquer, J.; Dávalos, A. Plasma betaamyloid 1-40 is associated with the diffuse small vessel disease subtype. Stroke, 2009, 40(10), 3197-3201.

[32] Goos, J.D.; Teunissen, C.E.; Veerhuis, R.; Verwey, N.A.; Barkhof, F.; Blankenstein, M.A.; Scheltens, P.; van der Flier, W.M. Microbleeds relate to altered amyloid-beta metabolism in Alzheimer's disease. Neurobiol. Aging, 2012, 33(5), 1011.e1-9.

[33] Carmichael, O.; Schwarz, C.; Drucker, D.; Fletcher, E.; Harvey, D.; Beckett, L.; Jack, C.R.Jr.; Weiner, M.; DeCarli, C.; Alzheimer's Disease Neuroimaging Initiative. Longitudinal changes in white matter disease and cognition in the first year of the Alzheimer disease neuroimaging initiative. Arch. Neurol., 2010, 67(11), 13701378.

[34] Viswanathan, A.; Greenberg, S.M. Cerebral amyloid angiopathy in the elderly. Ann. Neurol., 2011, 70(6), 871-880.

[35] Vemuri, P.; Weigand, S.D.; Przybelski, S.A.; Knopman, D.S.; Smith, G.E.; Trojanowski, J.Q.; Shaw, L.M.; Decarli, C.S.; Carmichael, O.; Bernstein, M.A.; Aisen, P.S.; Weiner, M.; Petersen, R.C.; Jack, C.R.Jr; Alzheimer's Disease Neuroimaging Initiative. Cognitive reserve and Alzheimer's disease biomarkers are independent determinants of cognition. Brain, 2011, 134(Pt 5), 1479-1492.

[36] Schneider, J.A.; Aggarwal, N.T.; Barnes, L.; Boyle, P.; Bennett, D.A. The neuropathology of older persons with and without dementia from community versus clinic cohorts. J. Alzheimers Dis., 2009, 18(3), 691-701.

[37] Kester, M.I.; Boelaarts, L.; Bouwman, F.H.; Vogels, R.L.; Groot, E.R.; van Elk, E.J.; Blankenstein, M.A.; van der Flier, W.M.; Scheltens, P. Diagnostic impact of CSF biomarkers in a local hospital memory clinic. Dement. Geriatr. Cogn. Disord., 2010 29(6), 491-497.

[38] Siderowf, A.; Xie, S.X.; Hurtig, H.; Weintraub, D.; Duda, J.; ChenPlotkin, A.; Shaw, L.M.; Van Deerlin, V.; Trojanowski, J.Q.; Clark, C. CSF amyloid \{beta\} 1-42 predicts cognitive decline in Parkinson disease. Neurology, 2010, 75(12), 1055-1061.

[39] Seppälä, T.T.; Koivisto, A.M.; Hartikainen, P.; Helisalmi, S.; Soininen, H.; Herukka, S.K. Longitudinal changes of CSF biomarkers in Alzheimer's disease. J. Alzheimers Dis., 2011, 25(4), 583-594.

[40] Chaudhuri, K.R.; Schapira, A.H. Non-motor symptoms of Parkinson's disease: dopaminergic pathophysiology and treatment. Lancet Neurol., 2009, 8(5), 464-474.

[41] Alves, G.; Larsen, J.P.; Emre, M.; Wentzel-Larsen, T.; Aarsland, D. Changes in motor subtype and risk for incident dementia in Parkinson's disease; a community-based studies on 171 subjects. Mov. Disord., 2006, 21(8), 1123-1130.

[42] Hely, M.A.; Reid, W.G.; Adena, M.A.; Halliday, G.M.; Morris, J.G. The Sydney multicenter study of Parkinson's disease: the inevitability of dementia at 20 years. Mov. Disord., 2008, 23(6), 837-844.

[43] Reid, W.G.; Hely, M.A.; Morris, J.G.; Loy, C. Halliday, G.M. Dementia in Parkinson's disease: a 20-year neuropsychological study (Sydney Multicentre Study). J. Neurol. Neurosurg. Psychiatry., 2011, 82(9), 1033-1037.

[44] Bibl, M.; Esselmann, H.; Lewczuk, P.; Trenkwalder, C.; Otto, M.; Kornhuber, J.; Wiltfang, J.; Mollenhauer, B. Combined Analysis of CSF Tau, $A \beta 42, A \beta 1-42 \%$ and $A \beta 1-40 \%$ in Alzheimer's Disease, Dementia with Lewy Bodies and Parkinson's Disease Dementia. Int. J. Alzheimers Dis., 2010, pii: 761571.

[45] Mollenhauer, B.; Trenkwalder, C.; von Ahsen, N.; Bibl, M.; Steinacker, P.; Brechlin, P.; Schindehuette, J.; Poser, S.; Wiltfang, J.; Otto, M. Beta-amlyoid 1-42 and tau-protein in cerebrospinal fluid of patients with Parkinson's disease dementia. Dement. Geriatr. Cogn. Disord., 2006, 22(3), 200-208.

[46] Halliday, G.M.; Song, Y.J.; Harding, A.J. Striatal $\beta$-amyloid in dementia with Lewy bodies but not Parkinson's disease. J. Neural Transm., 2011, 118(5), 713-719.

[47] Kalaitzakis, M.E.; Walls, A.J.; Pearce, R.K.; Gentleman, S.M. Striatal $A \beta$ peptide deposition mirrors dementia and differentiates DLB and PDD from other parkinsonian syndromes. Neurobiol. Dis., 2011, 41(2), 377-384. 
[48] Galpern, W.R.; Lang, A.E. Interface between tauopathies and synucleinopathies: a tale of two proteins. Ann. Neurol., 2006, 59(3), 449-458

[49] Tokuda, T.; Salem, S.A.; Allsop, D.; Mizuno, T.; Nakagawa, M.; Qureshi, M.M.; Locascio, J.J.; Schlossmacher, M.G.; El-Agnaf, O.M.A. Decreased $\alpha$-synuclein in cerebrospinal fluid of aged individuals and subjects with Parkinson's disease. Biochem. Biophys. Res. Commun., 2006, 349(1), 162-166.

[50] Aerts, M.B.; Esselink, R.A.; Abdo, W.F.; Bloem, B.R.; Verbeek, M.M. CSF $\alpha$-synuclein does not differentiate between parkinsonian disorders. Neurobiol. Aging, 2012, 33(2), 430.e1-3.

[51] Ohrfelt, A.; Grognet, P.; Andreasen, N.; Wallin, A.; Vanmechelen, E.; Blennow, K.; Zetterberg, H. Cerebrospinal fluid alphasynuclein in neurodegenerative disorders-a marker of synapse loss? Neurosci. Lett., 2009, 450(3), 332-335.

[52] Tateno, F.; Sakakibara, R.; Kawai, T.; Kishi, M.; Murano T. Alphasynuclein in the Cerebrospinal Fluid Differentiates Synucleinopathies (Parkinson Disease, Dementia With Lewy Bodies, Multiple System Atrophy) From Alzheimer Disease. Alzheimer Dis. Assoc. Disord., 2011, [Epub ahead of print].

[53] Mollenhauer, B.; Cullen, V.; Kahn, I.; Krastins, B.; Outeiro, T.F.; Pepivani, I.; Ng, J.; Schulz-Schaeffer, W.; Kretzschmar, H.A.; McLean, P.J.; Trenkwalder, C.; Sarracino, D.A.; Vonsattel, J.P.; Locascio, J.J.; El-Agnaf, O.M.; Schlossmacher, M.G. Direct quantification of CSF alphasynuclein by ELISA and first crosssectional study in patients with neurodegeneration. Exp. Neurol., 2008, 213(2), 315-325.

[54] Noguchi-Shinohara, M.; Tokuda, T.; Yoshita, M.; Kasai, T.; Ono, K.; Nakagawa, M.; El-Agnaf, O.M.; Yamada, M. CSF alphasynuclein levels in dementia with Lewy bodies and Alzheimer's disease. Brain Res., 2009, 1251, 1-6.

[55] Parnetti, L.; Chiasserini, D.; Bellomo, G.; Giannandrea, D.; De Carlo, C.; Qureshi, M.M.; Ardah, M.T.; Varghese, S.; Bonanni, L.; Borroni, B.; Tambasco, N.; Eusebi, P.; Rossi, A.; Onofrj, M.; Padovani, A.; Calabresi, P.; El-Agnaf, O. Cerebrospinal fluid Tau/ $\alpha$-synuclein ratio in Parkinson's disease and degenerative dementias. Mov. Disord., 2011, 26(8), 1428-1435.

[56] Shi, M.; Bradner, J.; Hancock, A.M.; Chung, K.A.; Quinn, J.F.; Peskind, E.R.; Galasko, D.; Jankovic, J.; Zabetian, C.P.; Kim, H.M.; Leverenz, J.B.; Montine, T.J.; Ginghina, C.; Kang, U.J.; Cain, K.C.; Wang, Y.; Aasly, J.; Goldstein, D.; Zhang, J. Cerebrospinal fluid biomarkers for Parkinson disease diagnosis and progression. Ann. Neurol., 2011, 69(3), 570-580.

[57] LeWitt, P.A.; Galloway, M.P.; Matson, W.; Milbury, P.; McDermott, M.; Srivastava, D.K.; Oakes, D. Markers of dopamine metabolism in Parkinson's disease. The Parkinson Study Group. Neurology, 1992, 42(11), 2111-2117.

[58] Lunardi, G.; Galati, S.; Tropepi, D.; Moschella, V.; Brusa, L.; Pierantozzi, M.; Stefani, A.; Rossi, S.; Fornai, F.; Fedele, E.; Stanzione, P.; Hainsworth, A.H.; Pisani, A. Correlation between changes in CSF dopamine turnover and development of dyskinesia in Parkinson's disease. Parkinsonism Relat. Disord., 2009, 15(5), 383-389.

[59] Pisani, V.; Stefani, A.; Pierantozzi, M.; Natoli, S.; Stanzione, P.; Franciotta, D.; Pisani, A. Increased blood-cerebrospinal fluid transfer of albumin in advanced Parkinson's disease. $J$ Neuroinflam., 2013, In press.

[60] Bishop, N.A.; Lu, T.; Yankner, B.A. Neural mechanisms of ageing and cognitive decline. Nature, 2010, 464(7288), 529-535.

[61] Crews, L.; Masliah, E. Molecular mechanisms of neurodegeneration in Alzheimer's disease. Hum. Mol. Genet., 2010, 19(R1), R12-R20.

[62] Snowdon, D.A.; Greiner, L.H.; Mortimer, J.A.; Riley, K.P.; Greiner, P.A.; Markesbery, W.R. Brain infarction and the clinical expression of Alzheimer disease. The Nun Study. JAMA, 1997, 277(10), 813-817.

[63] Ahmed, N.; Mandel, R.; Fain, M.J. Frailty: an emerging geriatric syndrome. Am. J. Med., 2007, 120(9), 748-753.

[64] Strawbridge, W.J.; Shema, S.J.; Balfour, J.L.; Higby, H.R.; Kaplan, G.A. Antecedents of frailty over three decades in an older cohort. $J$. Gerontol. B. Psychol. Sci. Soc. Sci., 1998, 53(1), S9-S16.

[65] Lebel, P.; Leduc, N.; Kergoat, M.J.; Latour, J.; Leclerc, C. Un modèle dynamique de la fragilité; in Campbell AJ ed: L'année gérontologique, Paris, Serdi, 1999, pp. 84-94.

[66] Binder, E.F.; Schechtman, K.B.; Ehsani, A.A.; Steger-May, K.; Brown, M.; Sinacore, D.R.; Yarasheski, K.E.; Holloszy, J.O Effects of exercise training on frailty in community-dwelling older adults: results of a randomized, controlled trial. J. Am. Geriatr. Soc., 2002, 50(12), 1921-1928.

[67] Gill, T.M.; Baker, D.I.; Gottschalk, M.; Peduzzi, P.N.; Allore, H.; Byers, A. A program to prevent functional decline in physically frail, elderly persons who live at home. N. Engl. J. Med., 2002, 347(14), 1068-1074.

[68] Stranahan, A.M.; Martin, B.; Maudsley, S. Anti-inflammatory effects of physical activity in relationship to improved cognitive status in humans and mouse models of Alzheimer's disease. Curr. Alzheimer Res., 2012, 9(1), 86-92.

[69] Ahlskog, J.E. Does vigorous exercise have a neuroprotective effect in Parkinson disease? Neurology, 2011, 77(3), 288-294. 